\title{
Status and Future of ADMX - the U.S. Microwave Cavity Axion Search Experiment
}

\author{
D. Kinion
}

This article was submitted to $4^{\text {th }}$ International Workshop on the Identification of Dark Matter (IDM2002), York, England, September 2-6, 2002

January 2, 2003

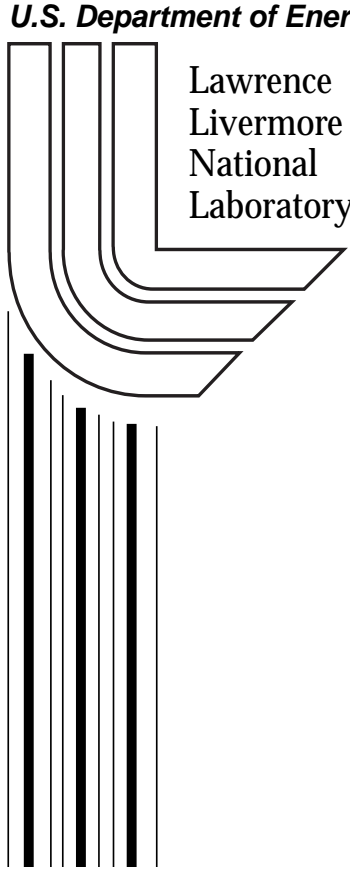




\section{DISCLAIMER}

This document was prepared as an account of work sponsored by an agency of the United States Government. Neither the United States Government nor the University of California nor any of their employees, makes any warranty, express or implied, or assumes any legal liability or responsibility for the accuracy, completeness, or usefulness of any information, apparatus, product, or process disclosed, or represents that its use would not infringe privately owned rights. Reference herein to any specific commercial product, process, or service by trade name, trademark, manufacturer, or otherwise, does not necessarily constitute or imply its endorsement, recommendation, or favoring by the United States Government or the University of California. The views and opinions of authors expressed herein do not necessarily state or reflect those of the United States Government or the University of California, and shall not be used for advertising or product endorsement purposes.

This is a preprint of a paper intended for publication in a journal or proceedings. Since changes may be made before publication, this preprint is made available with the understanding that it will not be cited or reproduced without the permission of the author.

This report has been reproduced directly from the best available copy.

Available electronically at http://www.doe.gov/bridge

Available for a processing fee to U.S. Department of Energy

and its contractors in paper from

U.S. Department of Energy

Office of Scientific and Technical Information

P.O. Box 62

Oak Ridge, TN 37831-0062

Telephone: (865) 576-8401

Facsimile: (865) 576-5728

E-mail: reports@adonis.osti.gov

Available for the sale to the public from

U.S. Department of Commerce

National Technical Information Service

5285 Port Royal Road

Springfield, VA 22161

Telephone: (800) 553-6847

Facsimile: (703) 605-6900

E-mail: orders@ntis.fedworld.gov

Online ordering: http://www.ntis.gov/ordering.htm

OR

Lawrence Livermore National Laboratory

Technical Information Department's Digital Library

http://www.llnl.gov/tid/Library.html

This work was performed under the auspices of the U.S. Department of Energy by the University of California, Lawrence Livermore National Laboatory under contract No. W-7405-Eng-48. 


\title{
STATUS AND FUTURE OF ADMX - THE U.S. MICROWAVE CAVITY AXION SEARCH EXPERIMENT
}

\author{
D. KINION \\ Lawrence Livermore National Laboratory, \\ 7000 East Ave., \\ Livermore, CA 94550, USA \\ E-mail: kinion1@llnl.gov
}

\begin{abstract}
I report on the status of the Axion Dark-Matter Experiment (ADMX), the microwave-cavity-based axion search underway at Lawrence Livermore National Laboratory. The ADMX collaboration includes LLNL, the University of Florida, and M.I.T., and has been in operation since February, 1996.
\end{abstract}

\section{Introduction}

To date, the most efficient method of searching for dark matter axions comprising the halo of our galaxy is the microwave cavity technique originally proposed by Sikivie ${ }^{1}$. In a static background magnetic field, axions will decay into single photons via the Primakoff effect. The energy of the photons is equal to the rest mass of the axion with a small contribution from its kinetic energy, hence their frequency is given by:

$$
h \nu=m_{a} c^{2}\left(1+O\left(10^{-6}\right)\right)
$$

At present, the allowed mass range of axions is $1-1000 \mu \mathrm{eV}$. At the lower end of this axion window, the frequency of the photons lies in the microwave regime. A high-Q resonant cavity, tuned to the axion mass will enhance the conversion process as well as serve as the detector for the converted photons. The expected signal power normalized to typical experimental parameters is ${ }^{1,2}$

$$
\begin{gathered}
P_{a \rightarrow \gamma} \approx 10^{-21} W\left(\frac{B}{7.7 T}\right)^{2}\left(\frac{V}{200 l}\right)\left(\frac{C}{0.65}\right) \\
\left(\frac{Q}{90000}\right)\left(\frac{\nu}{0.7 G H z}\right)\left(\frac{\rho_{a}}{\rho_{\text {halo }}}\right)
\end{gathered}
$$




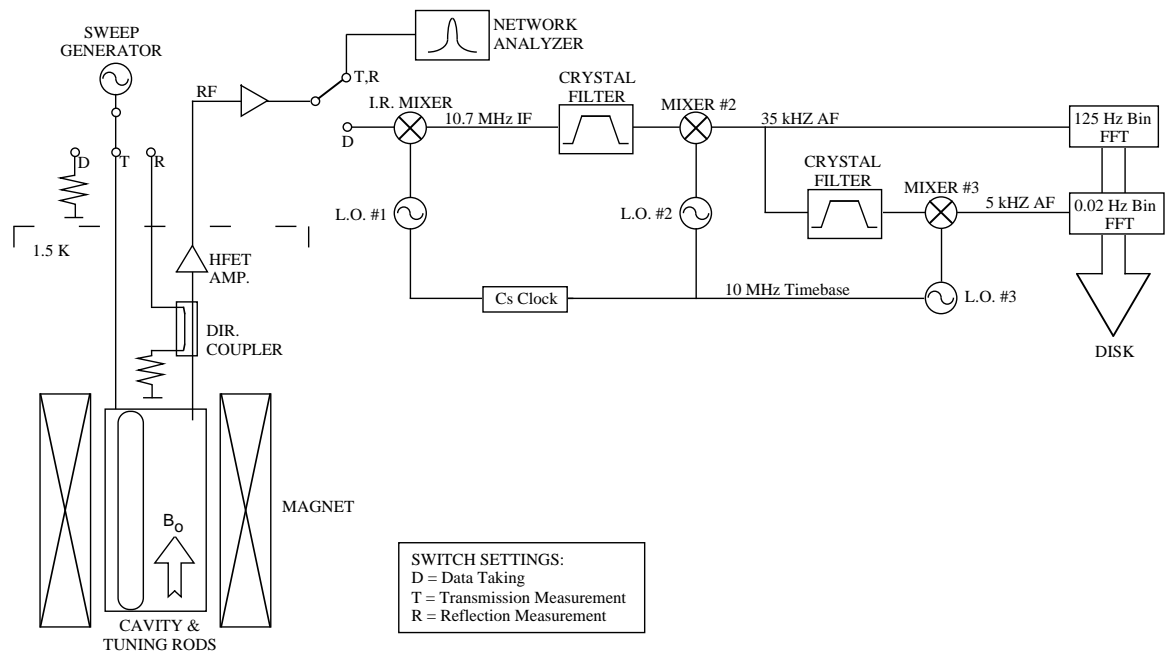

Figure 1. Schematic of the ADMX experiment.

where $\mathrm{B}$ is the background magnetic field, $\mathrm{V}$ is the cavity volume, $\mathrm{C}$ is a mode dependent form factor, $\mathrm{Q}$ is the loaded quality factor, $\mathrm{f}$ is the resonant frequency, and $\rho_{a}$ is the local halo axion density. For the parameters of this experiment, the power from KSVZ axions is typically $5 \times 10^{-22} \mathrm{~W}$.

Since the axion mass is unknown, the frequency of the cavity must be tunable. For a given signal-to-noise ratio (SNR) the scanning rate is:

$$
\frac{d \nu}{d t} \approx\left(\frac{25 M H z}{\text { month }}\right)\left(\frac{4}{S N R}\right)^{2}\left(\frac{6 K}{T_{s}}\right)^{2}
$$

where $T_{s}=T_{c}+T_{a}$ is the system noise temperature, specifically the sum of the physical temperature of the cavity $T_{c}$ and the noise temperature of the amplification chain $T_{a}$.

\section{Present Experiment and Results}

Figure 1 is a schematic of the ADMX apparatus showing the magnet, cavity, cryogenic amplifiers, and receiver electronics.

The magnet is a superconducting NbTi solenoid. It is a low current (224 A), high inductance $(533 \mathrm{H})$ design to maximize field stability, which serves to minimize eddy current heating of the cavity. The operating field at the center of the coil is typically $7.62-8.0 \mathrm{~T}$. 
The microwave cavity is a right-circular cylinder constructed from stainless steel and plated with ultra-high purity, oxygen-free copper. Annealing the cavity after plating increased the conductivity of the copper. The inside diameter is $50 \mathrm{~cm}$ and the length is $1 \mathrm{~m}$. Superfluid ${ }^{4} \mathrm{He}$ maintains the physical temperature of the cavity near $1.5 \mathrm{~K}$.

To maximize the form factor from (2), the cavity electric field should be parallel to the static, external magnetic field. The $\mathrm{TM}_{010}$ mode has the highest form factor $(C \approx 0.5-0.6)$, and is therefore used in the search. For this mode, the resonant frequency of the empty cavity is $460 \mathrm{MHz}$, and the unloaded Q is approximately 200,000 .

Moving a combination of metal and dielectric rods, running the full length of the cavity changes the resonant frequency. These rods can move from the center of the cavity to the wall. The single cavity accommodates two rods. Stepper motors with a resolution of $1.8^{\circ} /$ step followed by a gear reduction of 42000:1 control the rods. The final step size is approximately $80 \mathrm{~nm}$ and the resulting frequency tuning precision is approximately 500 $\mathrm{Hz}$ at a cavity frequency of $500 \mathrm{MHz}$.

The cryogenic amplifiers used in this search are double-balanced GaAs HFET amplifiers supplied by NRAO. ${ }^{3}$ The in situ measured noise temperatures range from $1.7-4.5 \mathrm{~K}$. Cascading two of these amplifiers achieves sufficient gain $(35 \mathrm{~dB})$ to render downstream noise contributions negligible.

The amplifiers are capacitively coupled to the cavity using a short length of low thermal conductivity semi-rigid coax. The strength of the coupling is varied by changing the insertion depth. Critical coupling is maintained throughout the run, meaning that on resonance the cavity presents a matched load to the first amplifier.

Figure 1 also shows the two major components of the room temperature electronics, the setup for measuring transmission through the cavity and the receiver electronics.

Before data is taken at a given frequency, a transmission measurement is made. For the measurement, power is fed through a second, very weakly coupled port in the cavity and the transmitted power is measured using a scalar network analyzer. A fit of the transmission curve to the sum of a Lorentzian and constant background determines the resonant frequency and Q.

The receiver is simply an extremely sensitive radio receiver with the cavity as the LC tank circuit. First, the $35 \mathrm{~dB}$ cryogenic amplification described earlier is followed by $35 \mathrm{~dB}$ of room-temperature post-amplification. Next, the signal passes through an image-reject mixer shifting the resonant 
frequency down to $10.7 \mathrm{MHz}$. From there, an oven-stabilized, eight-pole crystal filter sets the bandwidth of the measurement at $30 \mathrm{kHz}$. This filter also prevents image power from entering the subsequent mixing stages. Next, a second mixing stage shifts the center frequency to $35 \mathrm{kHz}$. This audio signal is then sent to both medium- and high-resolution search channels.

The medium resolution search channel consists of a commercial FFT spectrum analyzer. The sampling period of the analyzer is $80 \mathrm{msec}$, giving a frequency resolution of $125 \mathrm{~Hz}$. Each step involves averaging $10000 \mathrm{such}$ spectra, resulting in a 400 point power spectrum with $125 \mathrm{~Hz}$ bins. These data are coadded and the result searched for Maxwellian peaks a few bins wide (about $700 \mathrm{~Hz}$ ) characteristic of thermalized axions in the halo. ${ }^{4}$

An independent, high-resolution search channel operates in parallel to explore the possibility of fine-structure in the axion signal. ${ }^{5,6}$ The $35 \mathrm{kHz}$ signal passes through a six-pole crystal filter and third mixing stage to shift the center frequency to $5 \mathrm{kHz}$. During the 80 seconds that the medium resolution channel is averaging spectra, a PC based DSP takes a single 50 second spectrum and performs an FFT. The resulting frequency resolution is $20 \mathrm{mHz}$, about the limit imposed by the Doppler shift due to the earth's rotation. These data are searched for coincidences between different scans, as well as coincidences with peaks in the medium resolution data.

So far, no axion signal has been detected. Based on these results, we exclude at $90 \%$ confidence a KSVZ axion of mass between 2.5 and $3.3 \mu \mathrm{eV}$, assuming that thermalized axions comprise a major fraction of our galactic halo $\left(\rho_{a}=450 \mathrm{MeV} / \mathrm{cm}^{3}\right)$. This exclusion region and model predictions are shown in Figure 2. For more details see Ref. [7].

\section{Future Plans}

There are two major directions for this experiment to go in the future, up in frequency and down in sensitivity. Higher frequencies will require smaller cavities, which can be power combined to maintain effective use of the magnet volume. Work on this is already underway. Much higher frequencies may be achievable with a lattice of posts inside a larger cavity.

The ultimate goal of this experiment is to scan as much of the axion window as possible with DFSZ sensitivity. Since the expected power from DFSZ axion conversion is an order of magnitude lower than that from KSVZ axions it would take one hundred times longer to reach similar sensitivity. From Equation (3), the scanning rate goes as $T_{s}^{-2}$, therefore, an order 


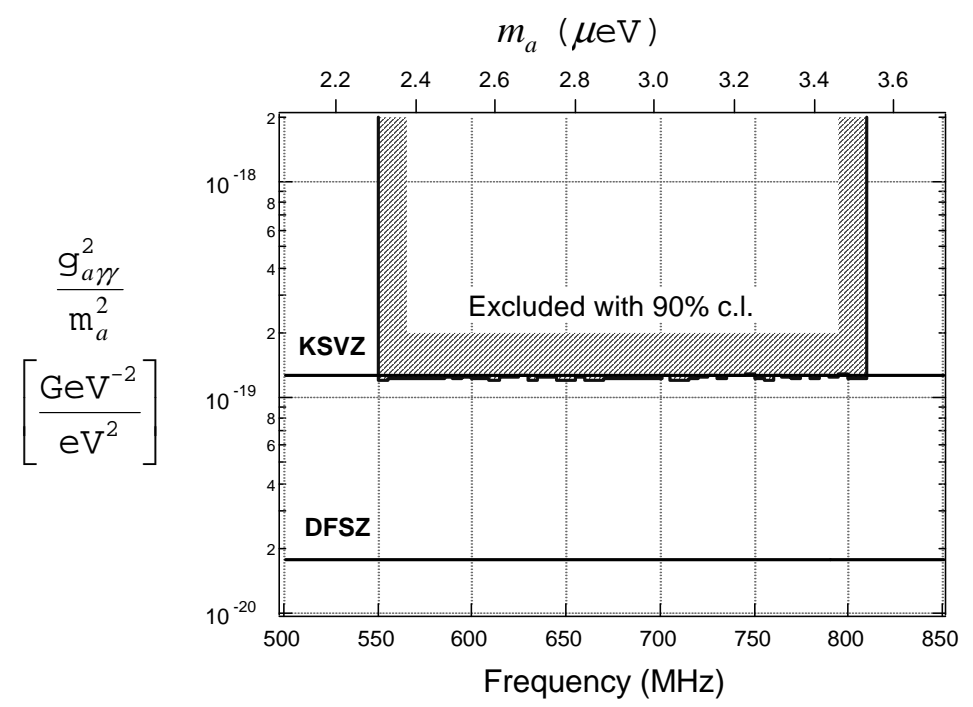

Figure 2. The range of $g_{a \gamma \gamma}^{2}$ excluded at $90 \%$ confidence by the 6 -bin search. Also shown are the KSVZ and DFSZ model predictions.

of magnitude reduction in system noise temperature would allow a scan at DFSZ sensitivity with the same rate as the present scan with KSVZ sensitivity. This is achievable with new dc SQUID based RF amplifiers which are already achieving noise temperatures within a factor of 3 of the Standard Quantum Limit. ${ }^{8}$

\section{Conclusion}

The ADMX experiment has been operating since February 1996 and has excluded KSVZ axions in the mass range 2.3 to $3.5 \mu \mathrm{eV}$ with greater than $90 \%$ confidence. The mass range below $2.3 \mu \mathrm{eV}$ is presently being scanned. An upgrade is being planned to incorporate SQUID amplifiers which should allow us to reach sensitivity to DFSZ axions.

\section{Acknowledgments}

This work was performed under the auspices of the U.S. Department of Energy by University of California, Lawrence Livermore National Laboratory under Contract W-7405-Eng-48. 


\section{References}

1. P. Sikivie, Phys. Rev. Lett. 51, 1415 (1983).

2. L. Krauss et al., Phys. Rev. Lett. 55, 1797 (1985).

3. E. Daw and R.F. Bradley, J. Appl. Phys. 82, 1925 (1997).

4. M.S. Turner, Phys. Rev. D 42, 3572 (1990).

5. P. Sikivie and J. Ipser, Phys. Lett. B 291, 288 (1992).

6. P. Sikivie et al., Phys. Rev. Lett. 75, 2911 (1995).

7. S. Asztalos et al., Phys. Rev. D 64, 092003 (2001).

8. Mück, M., J. B. Kycia, and J. Clarke, Appl. Phys. Lett. 78, 967 (2001). 